\title{
GRB 070714B-DISCOVERY OF THE HIGHEST SPECTROSCOPICALLY CONFIRMED SHORT BURST
} REDSHIFT

\author{
J. F. Graham ${ }^{1}$, A. S. Fruchter ${ }^{2}$, A. J. Levan ${ }^{3}$, A. Melandri ${ }^{4}$, L. J. Kewley ${ }^{5}$, E. M. Levesque ${ }^{5}$, M. Nysewander ${ }^{2}$, \\ N. R. TAnVir ${ }^{6}$, T. Dahlen ${ }^{2}$, D. Bersier ${ }^{4}$, K. Wiersema $^{6}$, D. G. Bonfield ${ }^{7}$, And A. MartineZ-Sansigre ${ }^{8}$ \\ ${ }^{1}$ Space Telescope Science Institute, Department of Physics and Astronomy, Johns Hopkins University, 3700 San Martin Drive, Baltimore, MD 21218, USA \\ ${ }^{2}$ Space Telescope Science Institute, 3700 San Martin Drive, Baltimore, MD 21218, USA \\ ${ }^{3}$ Department of Physics, University of Warwick, Coventry CV4 7AL, UK \\ ${ }^{4}$ Astrophysics Research Institute, Liverpool John Moores University, Twelve Quays House, Egerton Wharf, Birkenhead, CH41 1LD, UK \\ ${ }^{5}$ Institute of Astronomy, University of Hawaii, Honolulu, HI 96822, USA \\ ${ }^{6}$ Department of Physics and Astronomy, University of Leicester, University Road, Leicester LE1 7RH, UK \\ ${ }^{7}$ NASA Goddard Space Flight Center, 8800 Greenbelt Road, Greenbelt, MD 20771, USA \\ ${ }^{8}$ Max-Planck-Institute für Astronomie, Königstuhl 17, D-69117 Heidelberg, Germany \\ Received 2008 August 18; accepted 2009 April 6; published 2009 June 3
}

\begin{abstract}
We detect the optical afterglow and host galaxy of GRB 070714B. Our observations of the afterglow show an initial plateau in the light curve for approximately the first 5-25 minutes, and then steepening to a power-law decay with index $\alpha=0.86 \pm 0.10$ for the period between 1 and $24 \mathrm{hr}$ postburst. This is consistent with the X-ray light curve which shows an initial plateau followed by a similar subsequent decay. At late time, we detect a host galaxy at the location of the optical transient. Gemini Nod \& Shuffle spectroscopic observations of the host show a single emission line at $7167 \AA$ which, based on a griz JHK photometric redshift, we conclude is the $3727 \AA$ [O II] line. We therefore find a redshift of $z=0.923$. This redshift, as well as a subsequent probable spectroscopic redshift determination of GRB 070429B at $z=0.904$ by two other groups significantly exceeds the previous highest spectroscopically confirmed short burst redshift of $z=0.546$ for GRB 051221 . This dramatically moves back the time at which we know short bursts were being formed and suggests that the present evidence for an old progenitor population may be observationally biased.
\end{abstract}

Key words: gamma rays: bursts

\section{INTRODUCTION}

Although evidence of two classes of gamma-ray bursts (GRBs) has existed for over 25 years (Mazets et al. 1981; Norris et al. 1984), it was only with the work of Kouveliotou et al. (1993) 15 years ago that GRBs were widely recognized as being divided into two types based on duration and spectral slope: short and hard GRBs (SGRBs) and long and soft GRBs (LGRBs). Katz \& Canel (1996) then used the different $\left\langle V / V_{\max }\right\rangle$ (Schmidt et al. 1988) values between the SGRB and LGRB populations to claim a different distance distribution between the SGRB and LGRB populations, and to suggest that their formation mechanisms and thus their progenitors are likely different. They proposed that SGRBs were the product of compact object mergers (a possibility already noted earlier by, e.g., Blinnikov et al. 1984; Paczynski 1986; Goodman 1986; Eichler et al. 1989). More recently the discovery of short burst afterglows (see Hjorth et al. 2005b; Fox et al. 2005; Berger et al. 2005; Soderberg et al. 2006) has shown significant differences between the host galaxy populations of the two types.

Long bursts are predominantly associated with a particular galaxy type, faint blue irregular galaxies (Fruchter et al. 1999, 2006; Le Floc'h et al. 2003, 2002), and low metallicity environments in general (Fruchter et al. 2006; Stanek et al. 2007; Modjaz et al. 2008). Additionally, they show a strong preference for occurring not only in star-forming galaxies (Fruchter et al. 1999; Christensen et al. 2004; Le Floc'h et al. 2006) but also in the brightest, and hence likely most star-forming, regions of their hosts (Fruchter et al. 2006). This combined with them being frequently associated with subsequent type Ic supernova events (Stanek et al. 2003; Hjorth et al. 2003; Woosley \& Bloom
2006) has provided a coherent picture of the LGRB progenitor system.

With short bursts, a similar understanding is proving to be more elusive. While long bursts possess afterglows that are the most intense and among the most luminous astronomical phenomena (Kann et al. 2007; Bloom et al. 2008), short bursts tend to have fainter afterglows (Kann et al. 2008). Thus, absorption spectroscopy (which has provided a critical insight into the immediate progenitor environment of long bursts; e.g., Prochaska et al. 2006; Vreeswijk et al. 2007) of a short burst afterglow has, to date, not been successfully obtained (despite attempts; Stratta et al. 2007; Piranomonte et al. 2008). Until an SGRB afterglow absorption spectrum is obtained, almost all our knowledge of the burst environment, particularly the interstellar medium (ISM), must be derived from observations of the host galaxies associated with the bursts (Berger 2009).

Short burst host galaxies show a much greater diversity than those of long burst hosts. While the initial SGRB host associations were in elliptical galaxies (SGRB 050509B, Bloom et al. 2006; SGRB 050724, Berger et al. 2005) leading to claims that an old progenitor population was required, subsequent observations have suggested that this was due to small-number statistics. The occurrence of short bursts is now associated with all types of galaxies from an elliptical to a star-forming dwarf and is not obviously dependent on the host galaxy's (current) star formation (Gehrels et al. 2005; Berger et al. 2005). This is most likely indicative of a delay between progenitor system formation and burst occurrence (Nakar et al. 2006) and along with the absence of any correlating supernova emission (Hjorth et al. 2005a; Kann et al. 2008) makes single massive star core collapse an unlikely formation method. 
Due to the greater diversity of short burst host galaxies types, and the greater complexity in deriving a useful analysis of galaxy properties for some of these types, considerably greater complications are encountered in short burst host galaxy analysis requiring careful consideration of observational biases. Also, while the delay between the formation of short burst progenitors and the occurrence of the burst might allow binary progenitors to leave their host galaxies (see Belczynski et al. 2006), this should not be used as a panacea for explaining away host burst pairings with unacceptably large separations, especially considering that many SGRBs do not have bright hosts (Berger et al. 2007).

Compact object mergers, for example, neutron star-neutron star or neutron star-black hole (Eichler et al. 1989; Davies et al. 2005; Lee et al. 2005), are presently considered to be the preferred short burst progenitor candidate (see Gehrels et al. 2005; Bloom et al. 2006). Direct conclusive evidence will most likely come from gravitational wave detections of SGRB merger events, perhaps from the next generation of gravitational wave detectors. Frequency estimates for a LIGO II and Swift concurrent detection are in the range of 1 approximately every $3-$ 10 years (Seto 2005-for a neutron star-black hole and neutron star-neutron star merger, respectively). Until then, competing possibilities that satisfy the aforementioned criteria, such as millisecond pulsars with extremely strong magnetic fields (Usov 1992), collapse of neutron stars into black holes in binary systems (Dermer \& Atoyan 2006), and magnetar production via white dwarf-white dwarf mergers (Levan et al. 2006c), cannot be ruled out. However, host observations may provide the best opportunity to constrain various formation models.

Here, we report on the photometry and spectroscopy of our host galaxy of GRB 070714B, which lead us to conclude that the host is a moderately star-forming galaxy at a redshift of $z=0.923$ and suggesting that the present evidence for an old progenitor population may be, at least partially, observationally biased. The spectroscopy and redshift discussed here were originally reported in GCN 6836 (Graham et al. 2007).

\section{OBSERVATIONS AND DATA REDUCTION}

\subsection{Swift}

GRB 070714B was initially detected by the Burst Alert Telescope (BAT) on 2007 July 14 at 4:59:29 UT (Racusin et al. 2007b) on the NASA Swift spacecraft (Gehrels et al. 2004). The gamma ray emission consisted of several short spikes with a collective duration of $3 \mathrm{~s}$ followed approximately $20 \mathrm{~s}$ later by $50 \mathrm{~s}$ of softer emission. The main component also shows a small spectral lag (Norris et al. 2005). This emission is similar to previous short bursts including GRB 050724 (Barbier et al. 2007), and places this burst securely in the short category.

Rapid localization with the BAT instrument allowed the Swift satellite to slew its additional instruments onto the source. The onboard X-Ray Telescope (XRT) detected a new fading X-ray source 35 arcsec away from the final ground-processed BAT position (Racusin et al. 2007a). While the onboard UltraViolet Optical Telescope (UVOT) did not initially detect any new source within the XRT error circle, down to an unfiltered limit of 20.4 mag (Landsman \& Racusin 2007), a late-time reanalysis via coadding all prompt data detected a $4.5 \sigma$ optical transient with an unfiltered magnitude of $20.95 \pm 0.23$ (Landsman et al. 2007).

\subsection{Afterglow Follow-up}

Our first observations of GRB 070714B were undertaken with the Liverpool Telescope starting roughly 12 minutes after the burst. A series of short $10 \mathrm{~s} r$-band exposures followed by longer $120 \mathrm{~s}$ integrations in riz were obtained. We discovered an optical afterglow at R.A.: $03^{\mathrm{h}} 51^{\mathrm{m}} 22 \mathrm{~s} .2$, Decl.: $+28^{\circ} 17^{\mathrm{m}} 51^{\mathrm{s}} .4$ (J2000) with 0'.5 error (Melandri 2007), which places it within the XRT error circle (Racusin et al. 2007a). The afterglow was only marginally detected in the short exposures, and these were stacked to improve the accuracy of the photometry. Additional observations were obtained the following night at the William Herschel Telescope (WHT) in the $R$-band (Levan et al. 2007b). Subsequent observations with Gemini North detected no afterglow emission (see Section 2.3).

Photometry of the afterglow was taken using apertures with a radius equal to the FWHM of a stellar point source in each of the images. Our WHT $R$-band observations were photometered into the $r$ band by utilizing the photometric transformations of Jester et al. (2005); however, given the modest signal to noise in the detection, this transformation is not the dominant source of uncertainty. Absolute photometric calibration was obtained by using field stars to scale the zero point values to the Gemini field calibration exposures as described in Section 2.3. A log of the early photometry of the afterglow (and host contribution) is show in Table 1.

\subsection{Host Galaxy Optical Photometry}

Optical imaging of the host galaxy was obtained in $g, r, i$, and $z$ bands with the Gemini Multi-Object Spectrograph (GMOS) instrument on Gemini North between the nights of 2007 July 16 and 26. The number of individual exposures and nights of observation are given in Table 2. An individual exposure time of $300 \mathrm{~s}$ was used for all bands. With the exception of the $z$ band observations, this imaging was collected under nonphotometric conditions. Due to the narrow window between the object rising and dawn, optical observations were performed shortly before and often extending slightly past astronomical twilight, at airmasses just below and sometimes slightly above 2 , and had to be spread across several nights.

Photometric calibration observations were subsequently obtained of the object field and a standard field with GMOS on Gemini South on the night of September 25. Object field observations were performed with two $60 \mathrm{~s}$ exposures in all bands. Two standard exposures were taken in each band, encompassing four stars $(41,42,112$, and 115) in selected area 95 of Landolt (1992), of $8 \mathrm{~s}$ duration in $g, i$, and $z$ and $6 \mathrm{~s}$ in $r$. The final combined $i$ band image of the object field is shown in Figure 1.

Gemini optical imaging was reduced with the standard Gemini GMOS IRAF ${ }^{9}$ packages with fringing corrections applied in the $i$ and $z$ bands. The variable weather conditions and approaching dawn complicated the combination of the individual exposures into an optimal combined image.

Images in each band were scaled to a common intensity, aligned, and combined using a normalized weighting of the square of the inverse intensity scaling times the inverse sky median. Due to the near twilight nature of the observations the latter weighting was necessary to optimally correct for the worsening noise (due to increasing sky brightness) and

\footnotetext{
9 IRAF is distributed by the National Optical Astronomy Observatories, which is operated by the Association of Universities for Research in Astronomy, Inc., under cooperative agreement with the National Science Foundation.
} 
Table 1

Observations of the GRB 070714B Afterglow

\begin{tabular}{lccccc}
\hline \hline Date $($ UT) & $\Delta T$ (days) & Telescope & Band & Exposure Time & mag \pm error \\
\hline 14.21685 & 0.0088 & Liverpool & $r$ & $6 \times 10$ & $20.30 \pm 0.18$ \\
14.21917 & 0.0112 & Liverpool & $r$ & 120 & $20.14 \pm 0.11$ \\
14.22030 & 0.0123 & Liverpool & $i$ & 120 & $19.88 \pm 0.17$ \\
14.22473 & 0.0168 & Liverpool & $r$ & 120 & $20.28 \pm 0.25$ \\
14.22625 & 0.0183 & Liverpool & $r$ & 120 & $20.27 \pm 0.23$ \\
15.19236 & 0.9844 & WHT & $R$ & $8 \times 300$ & $24.11 \pm 0.21$ \\
\hline
\end{tabular}

Note. ( $r$ is the SDSS magnitude whereas $R$ is the Vega magnitude.) The host contribution has not been removed.

Table 2

Optical and IR Photometric Magnitudes of the Host Galaxy in Each Observed Band

\begin{tabular}{lcccccc}
\hline \hline Band & $\begin{array}{c}\text { Raw mag } \\
\pm \text { error }\end{array}$ & $\begin{array}{c}\text { Color Corrected } \\
\text { mag }\end{array}$ & $\begin{array}{c}\text { Dust Extinction } \\
\text { Corrected mag }\end{array}$ & $\begin{array}{c}\text { Date } \\
(\mathrm{UT})\end{array}$ & $\begin{array}{c}\text { Number of } \\
\text { Exposures }\end{array}$ & $\begin{array}{c}\text { Total Time } \\
(\mathrm{sec})\end{array}$ \\
\hline$g$ & $25.79 \pm 0.34$ & $25.81 \pm 0.34$ & $25.28 \pm 0.35$ & 17.6 & 5 & 1500 \\
$r$ & $24.90 \pm 0.21$ & $24.93 \pm 0.21$ & $24.54 \pm 0.22$ & 19.6 & $11^{\mathrm{a}}$ & 3300 \\
$i$ & $23.97 \pm 0.12$ & $23.96 \pm 0.12$ & $23.67 \pm 0.13$ & 22.6 & 8 & 2400 \\
$z$ & $24.01 \pm 0.13$ & $23.99 \pm 0.13$ & $23.78 \pm 0.13$ & 26.6 & 8 & 2400 \\
\hline$J$ & $22.27 \pm 0.12$ & & $22.14 \pm 0.12$ & 26.6 & 20 & 1200 \\
$H$ & $22.28 \pm 0.20$ & & $22.20 \pm 0.20$ & 25.6 & 17 & 1020 \\
$K$ & $21.13 \pm 0.13$ & & $21.08 \pm 0.13$ & 24.6 & 16 & 960 \\
\hline
\end{tabular}

Notes. Note that only the IR observations were performed under photometric conditions and the optical data was subsequently calibrated. ( $g, r, i$, and $z$ are SDSS magnitudes whereas $J, H$, and $K$ are Vega magnitudes). Errors include both the statical and systematic components.

a Three of these exposures were taken along with the $g$-band images on the 17 under comparatively poor conditions and comprise in total only $5 \%$ of the weight when combined into the final $r$-band image. (An additional two $r$-band images were taken on the 17 but were of such poor quality as to not be usable.)

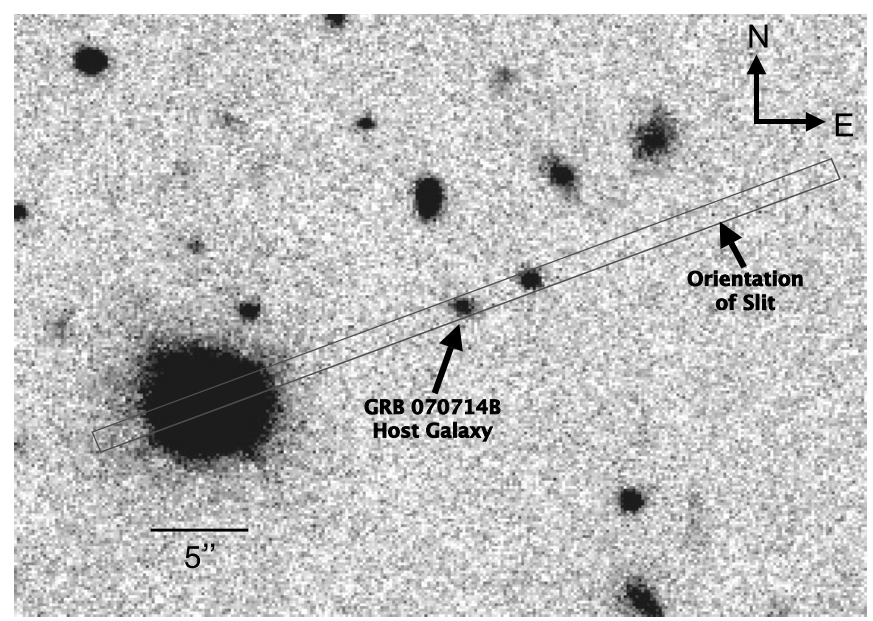

Figure 1. Combined $i$-band image of the GRB 070714B host and the surrounding field. The host and the slit orientation used in the spectroscopy are annotated.

corresponding change in signal to noise ratio. A small alteration was made to the "imcoadd" Gemini IRAF task to introduce the weight.

In order to calibrate this science imaging, the calibration exposures were similarly reduced but not combined. Sloan Digital Sky Survey (SDSS) magnitudes of the standard stars were used to determine zero point values of stars in each of the two standard calibration exposures in each band. (The SDSS survey magnitudes for the standards were used to maintain consistency in magnitudes systems with Gemini.) The average, for each band, was then used as the photometric zero point for the calibration images of the object field. Field stars were then used to determine the scaling between the calibration and science exposures in each band.
In the optical, a color correction between the GMOS-N filters and the SDSS filters is computed, using the values from Barr et al. (2005) in $g$ and $r$ and Jørgensen et al. (2005) in $r, i$, and $z$ (the $r$ corrections are identical in both sources), and is as expected negligible. A galactic extinction estimate of $E(B-V)=0.141 \mathrm{mag}$ from Schlegel et al. (1998) is then applied. The final magnitudes of the host galaxy in each band are shown in Table 2.

\subsection{IR Photometry}

We obtained $J H K$ near-infrared (NIR) imaging of the host galaxy of GRB 070714B with the Gemini South Near Infra-Red Imager (NIRI) instrument on the nights of 2007 July 24, 25, and 26. The details of the observations are given in Table 2. Single exposures were taken totaling 1 minute each in coadds of $5 \times$ $12 \mathrm{~s}, 6 \times 10 \mathrm{~s}$, and $5 \times 12 \mathrm{~s}$, in $J, H$, and $K$, for a total exposure time of 20,17, and 16 minutes, respectively. The images were taken under photometric conditions and low airmass.

Gemini NIR imaging was reduced with the standard Gemini NIRI IRAF package. Images were normalized by a flat field, dark and sky subtracted, aligned and combined using the standard NIRI reduction guidelines. In order to best account for variations in the sky, the images used for sky subtraction were based upon a rolling combination of exposures centered upon each image. The NIR images were calibrated to unsaturated field stars with magnitudes available in the Two Micron All Sky Survey (2MASS) catalogue, and were photometered in IRAF using a two FWHM aperture. The final magnitudes of the host galaxy in each band are shown in Table 2.

\subsection{Optical Spectroscopy}

Initial spectroscopic observations were obtained with the GMOS instrument on Gemini North on the night of the July 25. 
Due to the drop in detector sensitivity longward of $9250 \AA$, a central wavelength of $7250 \AA$ was selected yielding a spectral range of 5250-9250 A. Further spectroscopy was conducted on the night of September 13 with the central wavelength shifted out to $7750 \AA$ in an effort to extend the spectral coverage to include a line possibility discussed in Section 3.5.

The R400 grating offers a reasonable compromise between spectral resolution $\left(1.37 \AA\right.$ pixel $\left.^{-1}\right)$ and width of coverage (about $4000 \AA$ ) and was used both nights. A $50 \AA$ dither in wavelength was also added both nights to ensure continuous spectral coverage across chip gaps and allow for easy removal of other chip-based effects. Due to the abundance of skylines in the spectral range, the Nod \& Shuffle method was used. The first and second night of spectroscopy consisted of four and six 10 minute Nod \& Shuffle exposures, respectively.

In Nod \& Shuffle observing, only the central third of a typical long slit is opened and the telescope is repetitively offset (nodded) between two positions on the slit while the CCD charge wells are simultaneously moved (shuffled) between the illuminated central region and the nonilluminated upper and lower thirds of the CCD. These nonilluminated areas act as a storage region, buffering the data until the shutter is momentarily closed, the telescope offset, and the charge wells are shifted back into the illuminated region of the CCD. While the nod only requires that the object be moved off its location on the image and that sky land there instead, in practice the object is moved along the slit to another location on the image. Thus while one location is observing the object, the other is observing the sky and no time is spent solely gathering sky data. The result is a pair of images on the same CCD readout, both containing the object spectrum yet located in a different place with regard to the slit's field of view (FOV) on the image.

Nod \& Shuffle results in each CCD exposure being composed of multiple short exposures of the object interlaced with similar short exposures of the sky, each respectively stacked into separate images, each with an exposure time of half the total open shutter time of the Nod \& Shuffle exposure. This produces much more rapid temporal sampling of the sky than with separate successive exposures and a much lower noise than with a set of multiple separate images, each with its own readout thus eliminating the traditional tradeoff between the two concerns. While the time observed on the object remains the total open shutter time of the exposure, the drawback is in the closed shutter time lost to moving the telescope between the two offset positions. For a more detailed description of the Nod \& Shuffle process, see Cuillandre et al. (1994) and Glazebrook \& BlandHawthorn (2001) and for its use on Gemini, see Glazebrook \& the GDDS team (2003) and Abraham et al. (2003).

The individual spectroscopic exposures were reduced using the standard IRAF "Gemini.GMOS" Nod \& Shuffle packages and process. This is essentially the same as a conventional spectroscopic reduction except the two shuffled images on each exposure are subtracted from each other after bias subtraction and before flat fielding. This yields a single image for each exposure, each containing two spectra, one of which is inverted, separated by the nod distance. A custom Nod \& Shuffle dark was also used.

To optimize cosmic ray rejection for the relatively smaller number of Nod \& Shuffle spectra involved in this GRB host observation, we employ a deviation from the typical Nod \& Shuffle reduction process. Since each reduced Nod \& Shuffle exposure contains one positive and one inverted spectrum (as described above), an inverted copy of the images is created such

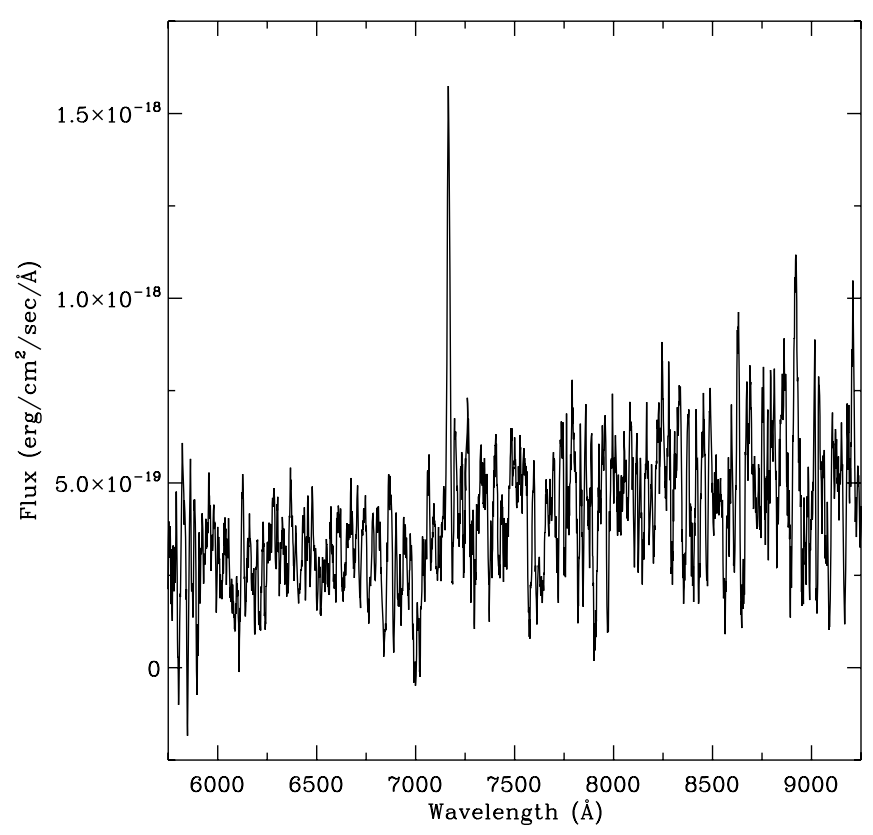

Figure 2. Gemini GMOS Nod \& Shuffle spectroscopy of the GRB 070714B host galaxy, smoothed with a 9 pixel boxcar function. In order to maintain a consistent depth of coverage, only the region of overlapping spectral coverage from the two central wavelengths is shown. Only a continuum and a single emission line at $7167 \AA$ was detected.

that (along with the originals) each spectrum is now present in an image without being inverted. This resulted in eight and twelve $300 \mathrm{~s}$ images for the 7250 and $7750 \AA$ central wavelengths, respectively. For each central wavelength an offset between the positive spectra is determined and the images are then combined using this offset in a single step, thus optimizing the cosmic ray rejection of the images being combined by doubling their number. (This is opposed to the conventional Nod $\&$ Shuffle reduction process were the reduced Nod \& Shuffle exposures are combined, with cosmic ray rejection, and the positive and inverted spectra on the combined output image are handled subsequently.) The two central wavelengths were then combined, weighted by their total exposure time.

Spectral extraction was performed with IRAF task "apall" using a 10 pixel wide aperture in the spatial direction. The continuum was too weak to allow automatic tracing of the aperture center. However a tracing of a bright star, also present in the slit, showed insignificant deviation in the spatial direction along the length of the spectra. Thus, a fixed center aperture was used.

The process yielded a spectrum with a spectral resolution of $1.37 \AA$ A per pixel and a spatial resolution of 0.15 arcsec per pixel. The spectrum is shown in Figure 2 and an enlargement of the region containing the spectral line in Figure 3. A single spectral line was observed at $7167 \AA$. Aside from a faint continuum, no other spectral features were detected in the 5150-9900 spectral range.

The spectrophotometric standard EG131 was observed immediately prior to the first epoch of spectroscopy and used to apply a relative flux calibration. However, because the spectroscopy and standard were observed under nonphotometric conditions, the relative flux calibrated spectrum was averaged (weighted by the product of the $i$-band filter transmissivity and the CCD spectral response) and then scaled such that this weighted average matches the calibrated $i$-band flux magnitude determined in Section 2.3. 


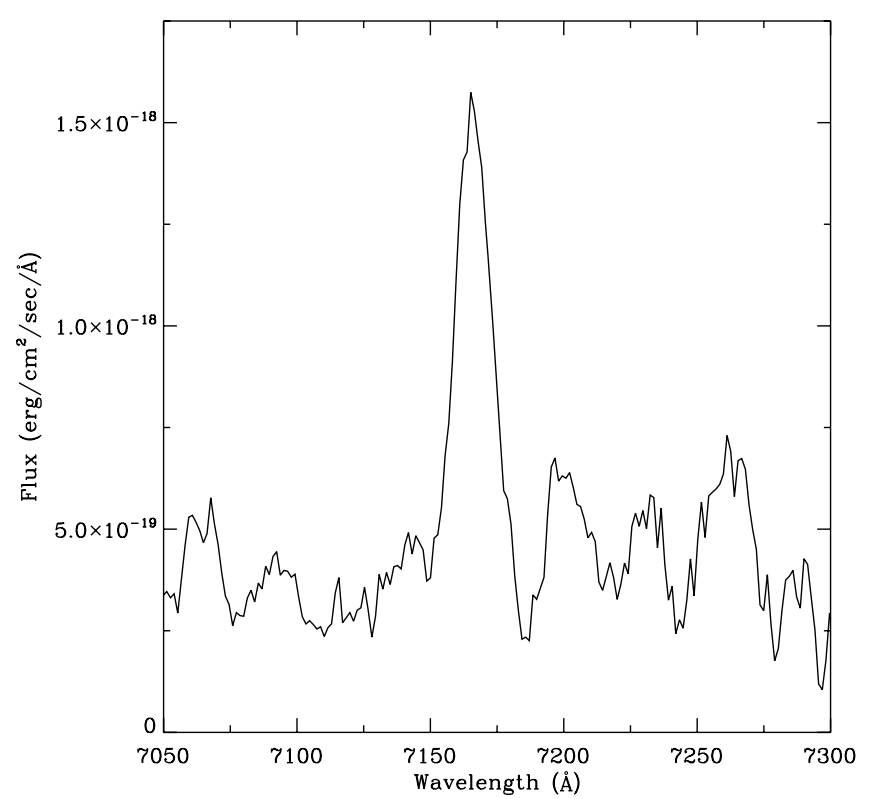

Figure 3. Enlarged view of the single emission line detected at $7167 \AA$ and the sounding region from our Gemini GMOS Nod \& Shuffle spectroscopy of the GRB 070714B host galaxy. Smoothed, as in Figure 2, with a 9 pixel boxcar function.

\subsection{NIR Spectroscopy}

NIR spectroscopy was performed on the host of GRB 070714B with NIRSPEC on the Keck II telescope on the night of October 23. The object was too faint to be visible on the acquisition camera and was acquired by placing the slit on a bright star and using a position angle that aligned the slit onto the object. Our observations consisted of eight 900 s exposures in the NIRSPEC-3 filter, using a 0.76 arcsec slit and giving a spectral coverage from 1.15 to $1.33 \mu \mathrm{m}$ and a spectral resolution of $2.33 \AA$ per pixel.

Individual NIRSPEC exposures were reduced using the standard procedure described in the online documentation (from the NIRSPEC manual). The object's placement on the slit was dithered between two locations (four exposures in each), so that the combined image from each placement could be subtracted from the other to remove sky features. However, this was complicated by the inadvertent placement of a field star on the slit at the sky subtraction position in one of the two settings. (These observations were unexpectedly obtained at the end of a night when it became clear that time otherwise planned could be used.) To compensate for this error, the typical process of subtracting the images and (then aligning and) adding the spectra was altered.

First, the sky effects were removed from individual exposures as best as possible via fitting the sky in the spatial direction with a high order polynomial and then subtracting off the fit. While this yielded a reasonably good removal of skylines and generated an image that was useable via human inspection, it was still inferior to a traditional sky subtraction with a twodimensional sky image. In particular, a few faint features remain in the spectral direction; these have the appearance of a faint spectral continuum, but move with the slit, and not the sky.

As no sources were detected in the individual spectra, the pixel offset needed to align the two dithered placements of the object on the CCD was determined from observations of another object using the same setup and dither value earlier in the night. The combined images for the two dithered placements were thus aligned and added.

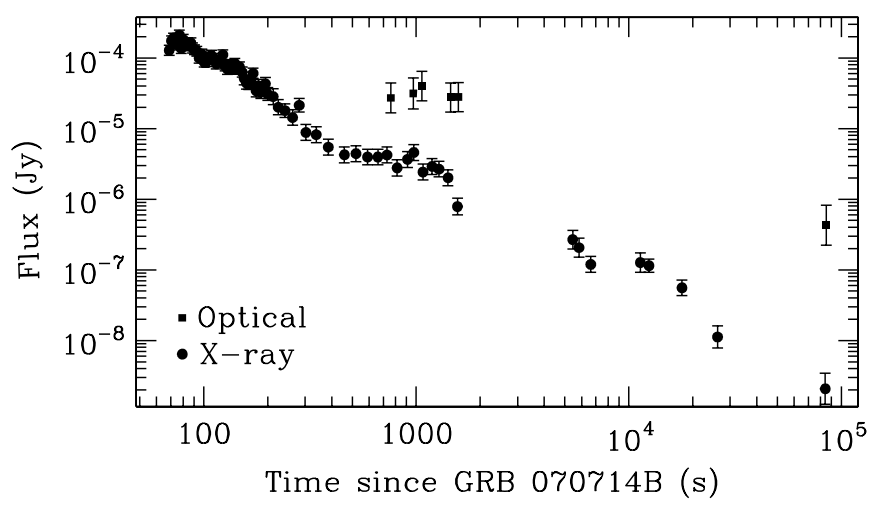

Figure 4. Optical observations of the GRB 070714B afterglow with the X-ray data overplotted. The host contribution has been removed. Note the coincident plateau in the optical and X-ray flux.

Additionally, another image was generated by reversing the direction of the offset when combining the images. Since all defects in the images are shared between the two dither placements, this creates an image without any object data but with an identical set of these defects, however also containing the bright star. Finally, this image is subtracted from the one described in the previous paragraph yielding a subtracted image identical to that given in the traditional method. However by blinking between this subtracted image and its operands, one can visually exclude the remaining sky features. No continuum or line for the object was detected.

To determine a rigorous upper limit value, the slit camera (SCAM) images were coadded for each dither position and then the coadded images were subtracted. The resulting images was inverted, shifted (to align the subsequently inverted negative features with their positive counterparts), and then coadded with its original self to generate a combined image. In addition to the bright star used for placing the slit, this uncovered two other stars on the final SCAM image. This image was then astrometrically compared with the Gemini GMOS i band final image to determine the location of the target galaxy and the additional nearby galaxy on the SCAM image (see Figure 1) and confirms that they were correctly placed on the slit.

A faint spectrum of the additional nearby galaxy described above was detected at the blue end of the spectrum. This, in addition to the spectrum of the bright star, was used to determine the relation between the objects on the SCAM image and the two-dimensional spectrum and interpolate the (expected) location of the object on the slit. A blind extraction was then used to generate a one-dimensional spectrum. Again, no continuum or line was apparent and based on the noise a $3 \sigma$ upper limit on the possible line flux of $4.4 \times 10^{-17} \mathrm{erg} \mathrm{s}^{-1} \mathrm{~cm}^{-2}$ was determined.

\section{ANALYSIS}

\subsection{Afterglow Follow-up}

Despite the low signal to noise, there is apparently little evidence of fading within the first Liverpool Telescope observations, which indicate that the afterglow decay was roughly flat, or decaying with $\alpha \sim 0.07 \pm 0.28$ (assuming a power-law decay of the form $F(t) \propto t^{-\alpha}$ ). This can be compared to the X-ray decay that shows a plateau during this period (see Figure 4).

Including our later time WHT observation, the optical decay rate becomes $\alpha=0.86 \pm 0.10$ (However, there is also a significant contribution from the host galaxy at this point which was subtracted but given the relatively small FOV of our WHT/ 
auxiliary port image, and the difference in the observed filter, a point spread function (PSF)-matched subtraction yields large residuals.) This can be compared to the X-ray decay over a similar time frame of $\alpha=1.73 \pm 0.11$ (Racusin et al. 2007a). While the optical points are roughly flat during the $\mathrm{x}$-ray plateau, suggesting that the two regimes were not entirely disconnected, the optical and x-ray behaviors clearly diverge at late times.

\subsection{Photometric Redshift}

Our optical and IR photometry also allows us to constrain the $7167 \AA$ A line identity via a photometric redshift determination. We calculated a photometric redshift probability distribution for the host galaxy using template fitting (Gwyn 1995; Mobasher et al. 1996). In this method, the observed photometry is matched to synthetic photometry derived using the filter throughputs and a library of galaxy spectral templates redshifted in the range $0<z<6$. The photometric redshift is derived by minimizing the $\chi^{2}$ value:

$$
\chi^{2}=\sum_{n=1}^{7}\left(\left[F_{\mathrm{obs}}^{n}-\alpha F_{\text {template }}^{n}\right] / \sigma^{i}\right)^{2},
$$

where the summation is taken over the seven filters available and $F_{\text {obs }}^{n}$ and $F_{\text {template }}^{n}$ are the observed and synthetic fluxes in band $n$, respectively. Finally, $\alpha$ is a normalization constant and $\sigma^{n}$ is the flux error in band $n$. The spectral templates used here consists of the E, Sbc, Scd, and Im templates from Coleman et al. (1980), together with two starburst templates from Kinney et al. (1996). Besides the best-fitting photometric redshift, this method also gives a redshift probability distribution, $P(z)$, where

$$
P(z) \propto \exp \left(-\chi^{2}\right) .
$$

The best-fitting photometric redshift and the relative probabilities for the three likely line possibilities discussed in Section 3.5 are calculated along with their respective best-fitting host galaxy spectral types. We find a best-fitting photometric redshift $z=0.83_{-0.20}^{+0.12}$ for an Scd-type galaxy. These results are shown in Table 3 and Figure 5.

Thus, the detected line is by a factor of 10 most likely the $3727 \AA$ [O II] line making the photometric redshift consistent with a GRB host galaxy at $z=0.923$. The best-fit spectral energy distribution (SED) is with an Scd-type galaxy template of magnitude $V=-19.7$. Figure 6 shows the measured photometry plotted over the redshifted template.

\subsection{Radial Velocity Curve and Correction}

A two-dimensional view of the $7167 \AA$ line (see Figure 7) shows an apparent shift in the line wavelength along the spatial direction due to the rotation of the galaxy. Fitting a radial velocity curve (see Figure 8 ) gives an estimated galactic rotation velocity of $110 \pm 20 \mathrm{~km} \mathrm{~s}^{-1}$. Assuming an $M_{V}=-19.7 \mathrm{Scd}$ galaxy as suggested by our photometric redshift fitting (see Section 3.2), and a rest-frame color term of $B-V=0.5$, applying the redshift dependent $B$-band Tully-Fisher relation in Böhm et al. (2004) gives a circular velocity of $83 \pm 20 \mathrm{~km} \mathrm{~s}^{-1}$. (Applying the local Tully-Fisher relation with the same color term gives a circular velocity of $121 \pm 15 \mathrm{~km} \mathrm{~s}^{-1}$.) Thus, the detected radial velocity curve is reasonably consistent with our photometric redshift fitting.

The radial velocity curve has the effect of spreading out the spectrum during one-dimensional extraction effectively

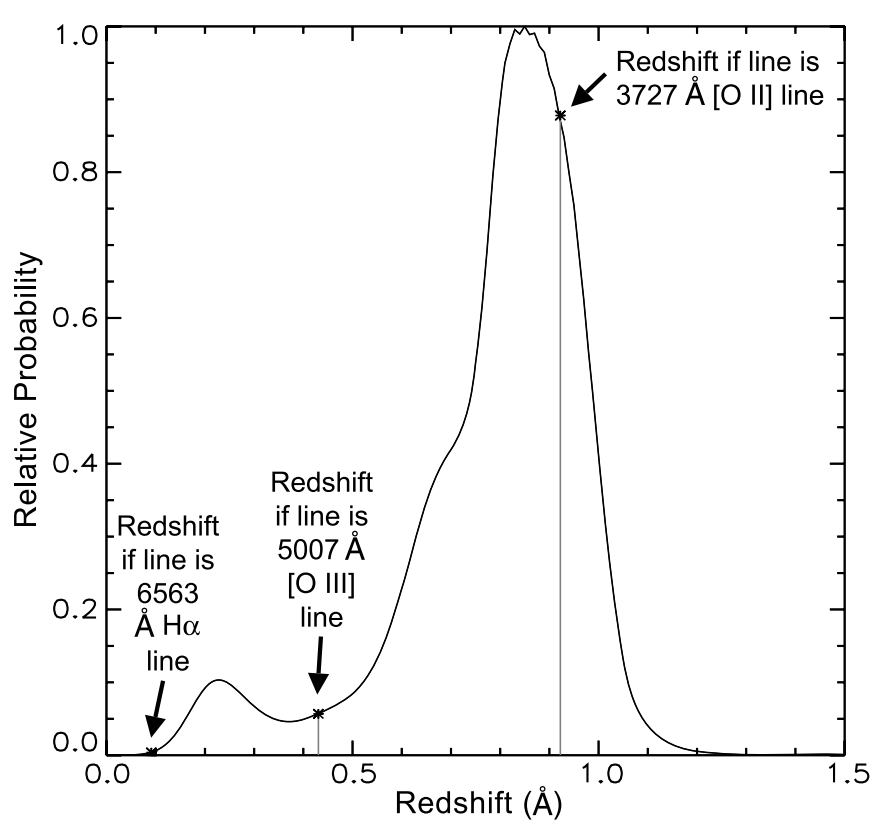

Figure 5. Photometric redshift relative probability distribution with the various line possibilities annotated.

decreasing the spectral resolution and correspondingly the achievable signal to noise. However, on the two-dimensional spectrum, the radial velocity curve is separated in the spatial direction; thus, this effect can be removed by shifting each row of the spectrum (in the spectral direction) such that spectral features line up in the spatial direction, effectively removing the rotation curve from the two-dimensional spectrum. Since the radial velocity curve closely approximates a difference of one spectral pixel for each spatial pixel, this shift was employed. The one-dimensional extraction was again performed with the IRAF "apall” task using identical parameters as previously employed (see Section 2.5). This countershifted spectrum is used subsequently and yields an estimated $15 \%$ improvement in signal to noise.

\subsection{Line Flux Measurement}

After smoothing, the continuum was fitted with a polynomial and the flux around the line was determined to be $6.39 \pm 0.30 \times$ $10^{-19} \mathrm{erg} \mathrm{s}^{-1} \mathrm{~cm}^{-2} \AA^{-1}$. Due to the spectrum being rather noisy, Gaussian fitting was thus unusually affected by the initial fitting parameters. While this fitting yielded values consistent with the line flux determined subsequently (using a method that makes no assumption on the lines shape and width) estimates of the error on the determined flux values were an unacceptably high $20 \%-30 \%$ of the line flux.

In order to obtain a more robust measure of the flux, a curve of growth technique was used in which the spectrum is boxcar summed with increasing widths and the resultant highest value pixel taken as the flux. With this method, the flux will be underestimated until a sufficient width is achieved to encompass all line flux and thereafter higher widths will cause the flux measurements to oscillate, to increasing degree, about the true line flux from the increasing noise added via the addition of nonline pixels into the smoothing. This approach is equivalent to the "curve-of-growth" technique used in photometry.

An optimal smoothing of 17 pixels yielded a flux with a curve-of-growth fitting error of $1.664 \times 10^{-18} \mathrm{erg} \mathrm{s}^{-1} \mathrm{~cm}^{-2}$. An additional error of $1.855 \times 10^{-19} \mathrm{erg} \mathrm{s}^{-1} \mathrm{~cm}^{-2}$ pixel ${ }^{-1}$ 


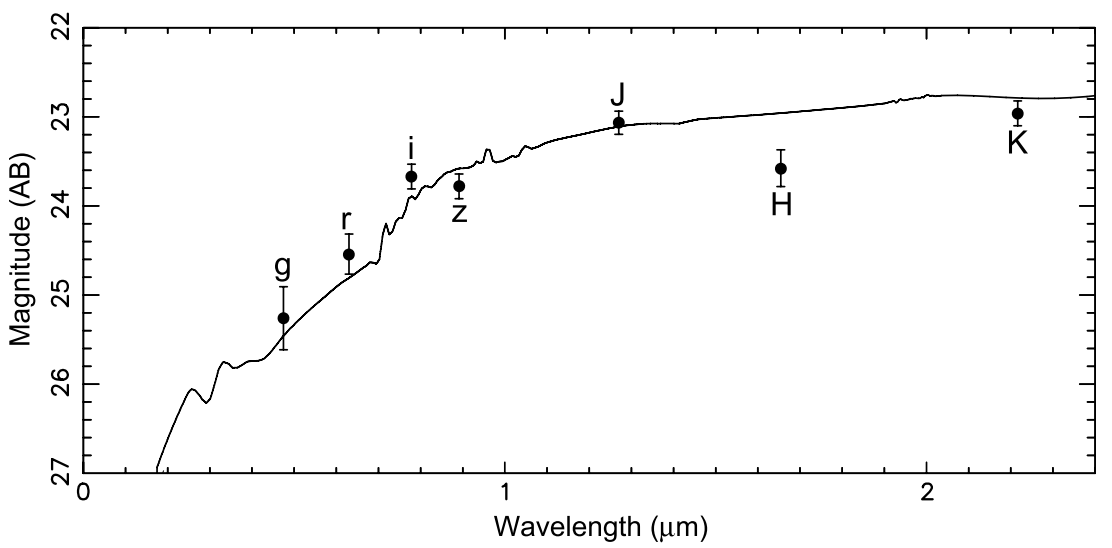

Figure 6. SED template of the $z=0.923$ fit (solid line) with the measured photometric values over-plotted. (The measured photometric values have been converted to $\mathrm{AB}$ magnitudes.)

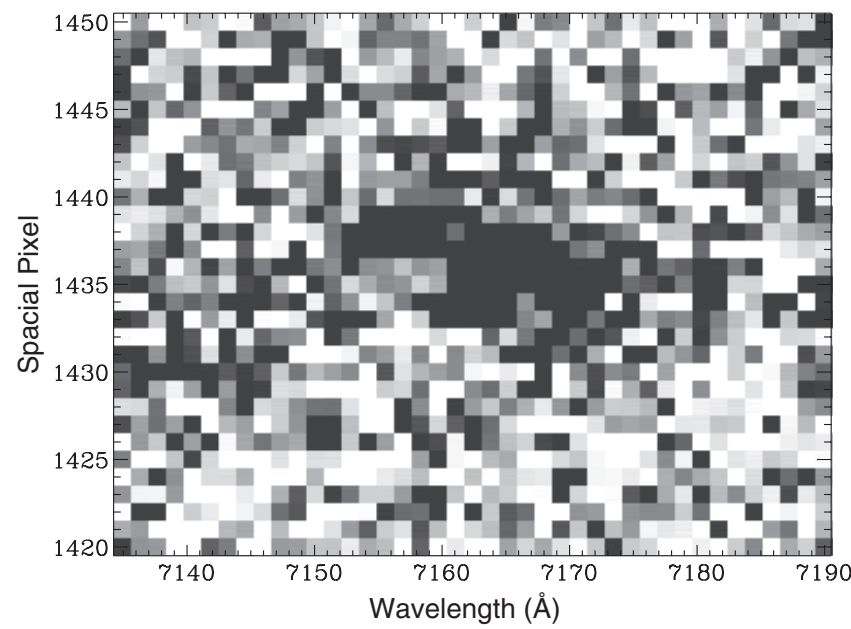

Figure 7. Two-dimensional spectra image of the $7167 \AA$ line. Note that the line has a radial velocity skew.

is calculated from the standard deviation of the smoothed spectrum around the line from the continuum. The flux of the $7167 \AA$ line (in the observer frame) is thus determined to be $2.7 \pm 0.36 \times 10^{-17} \mathrm{erg} \mathrm{s}^{-1} \mathrm{~cm}^{-2}$ yielding a $\mathrm{S} / \mathrm{N}$ of 7.69 and an (galactic rest frame) equivalent width of $-22.3 \pm 2.9 \AA$ (assuming that the line is the 3727 [O II] line).

\subsection{Spectroscopic Constraints}

In addition to photometric redshift fitting, it is possible to consider constraints on the identity of the detected line based on the flux limits for nondetected lines. Given the range of continuous spectral coverage obtained, there are three reasonable candidates for the single observed spectral line at $7165 \AA$ : the $3727 \AA\left[\mathrm{O}_{\text {II }}\right]$ line placing the object at a redshift of $z=0.92$, the $5007 \AA[\mathrm{O}$ III] line placing the object at a redshift of $z=0.43$, and the $6563 \mathrm{H} \alpha$ line placing the object at a redshift of $z=0.09$. The latter possibility is highly unlikely (see Table 3) and can be excluded given the color of the object alone and thus is not given further consideration. The $4959 \AA$ [O II] line is also notable but not a candidate due to its flux being quantum mechanically pegged at about a third of the flux of the $5007 \AA$ [O III] line.

Assuming that the $7167 \AA$ spectral feature is the $3727 \AA$ [O II] line, the $5007 \AA$ [O III] line would be expected at a detector wavelength of $9628 \AA$. While the GMOS sensitivity is quite
Table 3

Photometric Redshift Relative Probabilities for Various Line Possibilities

\begin{tabular}{lcccc}
\hline \hline $\begin{array}{l}\text { Line } \\
\text { Matched }\end{array}$ & $\begin{array}{c}\text { Redshift } \\
(z)\end{array}$ & $\begin{array}{c}\text { Relative } \\
\text { Probability }\end{array}$ & $\begin{array}{c}\text { Galaxy } \\
\text { Type }\end{array}$ & $\begin{array}{c}\text { Absolute } \\
\text { Mag (V) }\end{array}$ \\
\hline Pure Photometric Fit & 0.83 & 1 & Scd & -19.4 \\
$6563 \AA$ H $\alpha$ & 0.09 & 0.004 & Sbc & -13.8 \\
$5007 \AA$ [O III] & 0.43 & 0.057 & Scd & -17.8 \\
$3727 \AA$ [O II] & 0.92 & 0.88 & Scd & -19.7 \\
\hline
\end{tabular}

poor beyond $9250 \AA$, the expected position was included in the spectral range of the September 13 spectroscopy. No line was positively detected; however, a feature was seen at the expected wavelength with an approximate flux of $5 \times 10^{-17} \mathrm{erg}$ $\mathrm{s}^{-1} \mathrm{~cm}^{-2}$ which, while not typical of, is consistent with other noise features in its respective region. A flux upper limit of $6.7 \times 10^{-18} \mathrm{erg} \mathrm{s}^{-1} \mathrm{~cm}^{-2}$ can be placed on the $5007 \AA$ [O III] line. This yields a lower limit on the $3727 \AA$ [O II] to $5007 \AA$ [O III] line flux ratio of $0.40 \pm 0.19$ in the $z=0.92$ case. Similarly, assuming that the $7167 \AA$ spectral feature is the $5007 \AA$ [O III] line, the $3727 \AA$ [O II] line would be expected at a detector wavelength of $5335 \AA$ (within the spectral range of the July 25 spectroscopy). No line was detected; however, a flux limit of $4.0 \times 10^{-17} \mathrm{erg} \mathrm{s}^{-1} \mathrm{~cm}^{-2}$ can be placed on the nondetected $3727 \AA$ [O II] line. This yields an upper limit on the $3727 \AA$ [O II] to $5007 \AA$ [O III] line flux ratio of $1.48 \pm$ 0.31 in the $z=0.43$ case. Since the $3727 \AA$ [O II] to $5007 \AA$ [O III] line flux ratio is degenerately dependent on the extinction, ionization parameter, and metallicity of the galaxy, a wide range of ratios have been observed with values ranging from 0.02 to 25 (Kewley \& Dopita 2002) and without additional information on the galaxy the permissible range cannot be constrained (galaxy type is not particularly constraining). Thus, no exclusion can be placed on either possibility based on the $3727 \AA$ [O II] to $5007 \AA$ [O III] line flux ratio.

A more constraining limit on the $5007 \AA$ [O III] line case is that the $4959 \AA$ [O III] line would be expected at $7098 \AA$. Again, no line was detected; however, a flux limit of $6.8 \times 10^{-18} \mathrm{erg}$ $\mathrm{s}^{-1} \mathrm{~cm}^{-2}$ can be placed on the nondetected $4959 \AA$ [O III] line. Since the $4959 \AA$ [O III] line and the $5007 \AA$ [O III] line are both the result of spontaneous forbidden transitions from the $2 s^{2} 2 p^{2}{ }^{1} D_{2}$ state (to $2 s^{2} 2 p^{2}{ }^{3} P_{1}$ and $2 s^{2} 2 p^{2}{ }^{3} P_{2}$, respectively), they have a quantum mechanically fixed flux ratio of 1:3.01 (Storey \& Zeippen 2000) corresponding to a flux intensity ratio of 1:2.98 (Storey \& Zeippen 2000). This is in reasonable 


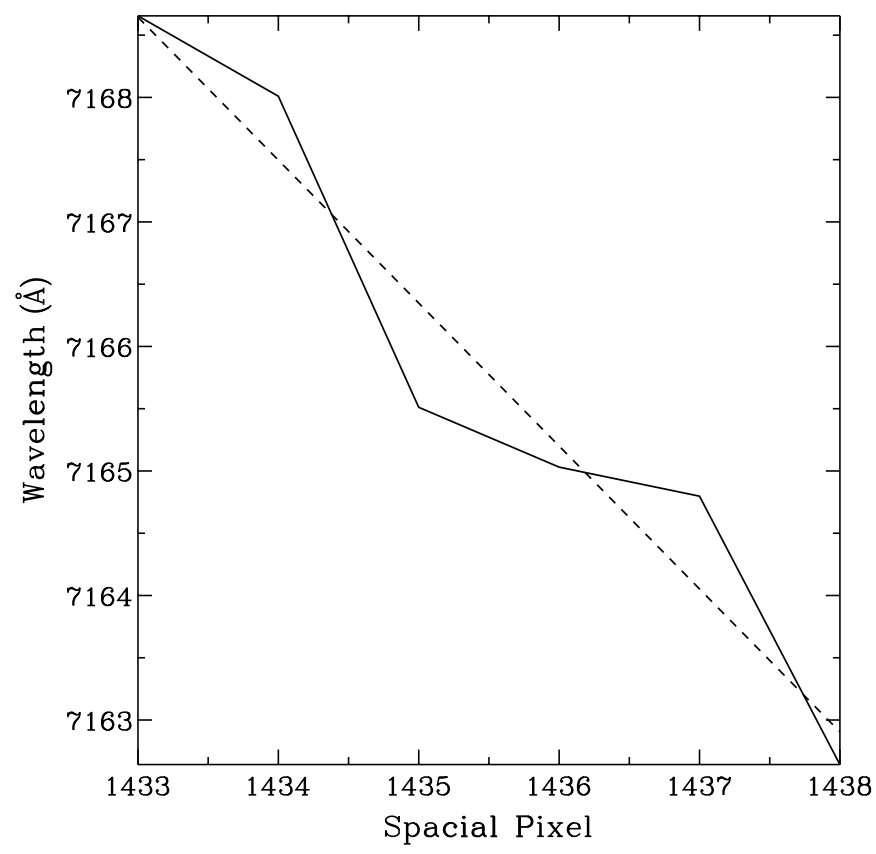

Figure 8. Radial velocity plot of the $7167 \AA$ line. The dotted line shows the fitted radial velocity.

agreement with the observed galactic line flux ratio of 1:2.953 \pm 0.014 (Dimitrijević et al. 2007). Thus, the expected flux of the $4959 \AA$ [O III] line is $9.06 \pm 1.21 \times 10^{-18} \mathrm{erg} \mathrm{s}^{-1} \mathrm{~cm}^{-2}$ and quite well constrained. This expected flux marginally exceeds the observed flux limit.

For the $3727 \AA[\mathrm{OII}]$ line case, NIR spectroscopy was performed in an effort to detect the $\mathrm{H} \alpha$ line shifted out to $1.26 \mu \mathrm{m}$. No line was detected; however, a flux limit of $4.4 \times 10^{-17} \mathrm{erg} \mathrm{s}^{-1} \mathrm{~cm}^{-2}$ can be placed on the nondetected line. Due to the expected observer frame $\mathrm{H} \alpha$ wavelength being close to the $J$-band central wavelength, we can make a crude estimate on the continuum flux based on our $J$-band IR imaging. This gives an estimated continuum flux of $3.7 \times 10^{-19} \mathrm{erg} \mathrm{s}^{-1}$ $\mathrm{cm}^{-2} \AA^{-1}$ and a corresponding approximate upper limit on the $\mathrm{H} \alpha$ (galactic rest frame) equivalent width of $62 \AA$. This yields a lower limit on the $3727 \AA$ [O II] to $\mathrm{H} \alpha$ ratio in flux of 0.45 and an equivalent width of 0.36 which, given that a majority of galaxies are within these constraints, makes the nondetection of the $\mathrm{H} \alpha$ line perfectly reasonable.

Thus, from the spectroscopic data alone, the $3727 \AA$ [O II] line is marginally favored based on the exclusion of the $5007 \AA$ [O III] case from the nondetection of the $4959 \AA$ [O III] line at the quantum mechanically required flux ratio. However, in concert with photometric redshift fitting that strongly favors $3727 \AA$ [O II] as the line, this can be reasonably assumed to be the correct identification and yields a redshift of $z=0.923$ for the host galaxy.

\section{CONCLUSIONS}

Initial Swift observations put GRB 070714B securely in the short burst category. Observations with the Liverpool Telescope detected an optical afterglow with an initial plateau for the first 5-25 minutes, then subsequent decay steepening to $\alpha=$ $0.86 \pm 0.10$ for the remaining first $24 \mathrm{hr}$ postburst. This is consistent with the X-ray light curve, which also shows an initial plateau followed by a concurrent decay of $\alpha=1.73 \pm 0.11$ (Racusin et al. 2007a), suggesting that the X-ray and optical regimes were not entirely disconnected.
We also detect a host galaxy with an angular extent that includes the location of the optical transient $\left(0^{\prime \prime} 4\right.$ from the host galaxy center). Our Gemini Nod \& Shuffle spectroscopic observations of the host show a single emission line at $7167 \AA$, which, based on a photometric redshift from our griz JHK multiband optical and IR photometry, implies that this can only be the $3727 \AA\left[\mathrm{O}_{\text {II }}\right]$ line. This places the host at a redshift of $z=0.923$, the highest spectroscopically confirmed redshift for a short burst.

Photometric fitting shows the host to possess a type Scd stellar population and its luminosity, color, and radial velocity are all consistent with an Scd and are in fact quite similar to M33. The host's diameter, about 7-8 kpc, is notably only about half of M33's; however, given the redshift of the object $(z=0.92)$ and that grand design spirals only emerge at around $z$ of 1 , this difference in angular size does not seem that problematic. Planned Hubble Space Telescope (HST) imaging should allow us to tell whether the similarity between colors and velocity extends to morphology.

Subsequent to our discovery of the redshift of SGRB 070714B, the host galaxy of SGRB 070429B was observed with Keck to have a spectral line at $7091 \AA$. This, the observers suggest, was the $3727 \AA$ [O II] line, placing it at a redshift of $z=0.904$ (Perley et al. 2007; Cenko et al. 2008). However, in the absence of either a photometric redshift or other spectral features, it is hard to estimate the likelihood that this line identification is correct. Prior to these discoveries, the highest spectroscopically confirmed short burst redshift was SGRB 051221 at $z=0.546$ (Soderberg et al. 2006).

A number of observers have obtained the redshifts of bright galaxies found within XRT error circles, and have suggested that these may be used as the redshifts of the bursts (Berger et al. 2007). However, Cobb \& Bailyn (2008) have shown that if an $I<21.5$ magnitude galaxy is detected in an XRT error circle, there is a $50 \%$ chance that it is due to random alignment of an unrelated galaxy. In contrast, they expect a contamination rate of only $1 \%$ based on the detection of an optical transient within the angular extent of a galaxy. Indeed, even a radius of $5^{\prime \prime}$ (consistent with many claimed associations and typical of the pre-UVOT-enhanced XRT position error circles) would have a greater than $60 \%$ chance of containing a random galaxy as bright as the host of GRB 070714B based on Hubble Deep Field (HDF) galaxy counts (Williams et al. 1996). Thus, only in cases such as GRBs 070714B and 070429B where the burst has an optical afterglow within the angular extent of a galaxy can the host association be made with very little chance of confusion (Levan et al. 2007a). We are fortunate in the case of GRB 070714B to be able to further strengthen our single-line redshift measurement by a highly constraining photometric redshift.

While GRB 070714B is now the short burst with the highest spectroscopic redshift, and alone extends examples of short burst formation throughout the recent half of the Universe, there are other short bursts that may well be more distant. Berger et al. (2007) has pointed out that there are no bright galaxies in the XRT error circles of a number of bursts, suggesting that at least some of these are at redshifts $z>1$ and indeed there are cases like GRB 060121 (Levan et al. 2006a) where deep observations have turned up faint hosts not initially detected. Furthermore, photometric observations of the afterglow and host of GRB 060121 suggest that it was probably above $z \sim 1$ and may have been at $z \sim 4.5$ (Donaghy et al. 2006; de Ugarte Postigo et al. 2006; Levan et al. 2006b). Recent additional HST and Spitzer observations of the host of SGRB 060121 may produce a more 


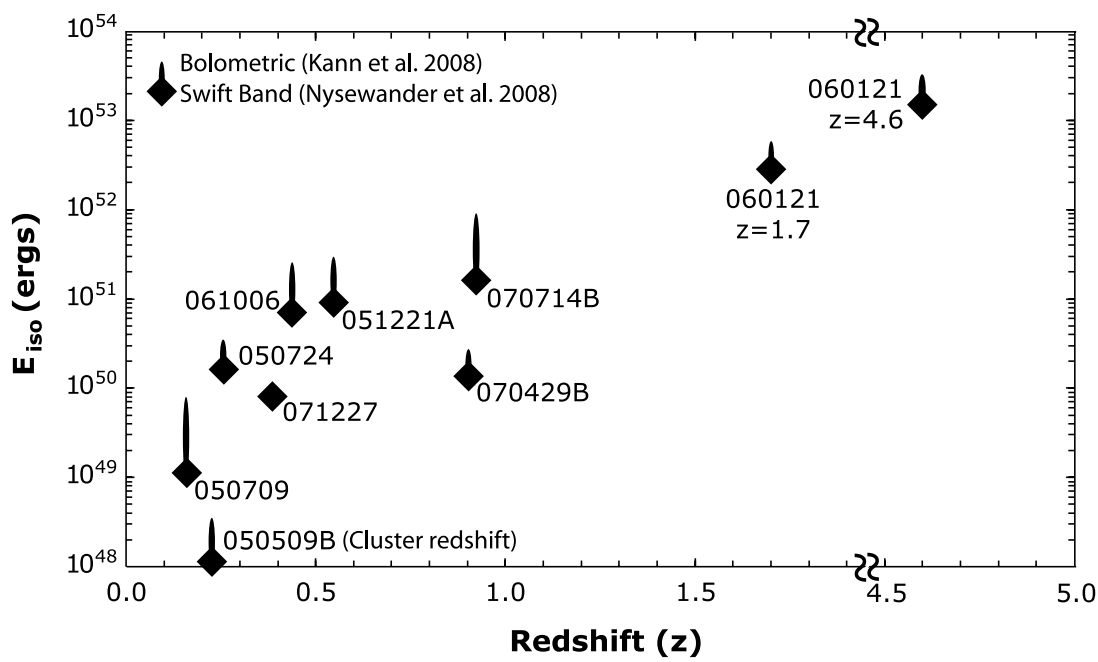

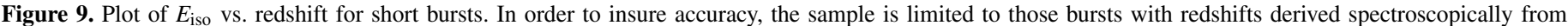

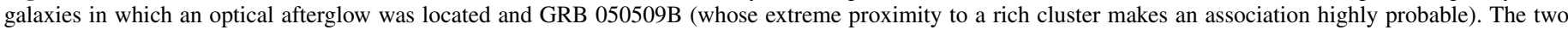

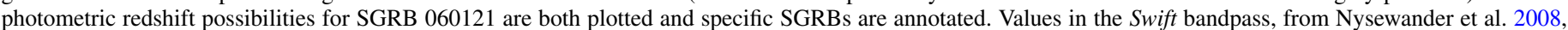

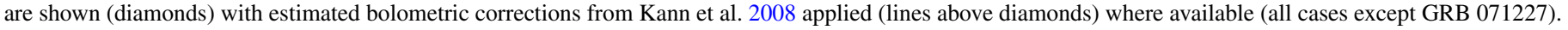

reliable photometric redshift estimate (A. J. Levan et al. 2009, in preparation). Additionally, the possibility that the highest redshift GRB (GRB 080913 at $z=6.7$ ) might be categorized as a short burst would push short burst formation even into the Universe's first billion years (Belczynski et al. 2008; Greiner et al. 2009; Zhang et al. 2009).

From our redshift and the observed burst fluence (of $7.2 \times$ $10^{-7} \mathrm{erg}^{-2}$ from GCN 6623; Barbier et al. 2007), we calculate an isotropic energy release (Eiso) for SGRB 070714B of $1.61 \pm 0.20 \times 10^{51} \mathrm{erg}$ (in the Swift bandpass pass). Kann et al. (2008) (using the method outlined in Bloom et al. 2001) estimated a bolometric isotropic energy release (Eiso) of $9.8_{-2.0}^{+4.0} \times 10^{51} \mathrm{erg}$, which is in reasonable agreement with a $15-2000 \mathrm{keV}$ band value of $8.3_{-1.3}^{+2.9} \times 10^{51} \mathrm{erg}$ calculated from a joint Swift + Suzaku-WAM spectral analysis fluence value of $3.7_{-0.6}^{+1.3} \times 10^{-6} \mathrm{erg} \mathrm{cm}^{-2}$ (Ohno et al. 2007). This places SGRB 070714B about an order of magnitude more luminous than the median short burst yet still about an order of magnitude below the luminosity of the median long burst (using the data compiled in Nysewander et al. 2008). SGRB 070714B has almost twice the energy release of the previous highest redshift short burst SGRB 051221 and makes it the most luminous short burst (from the sample of those with spectroscopic redshifts) yet seen (Nysewander et al. 2008; Kann et al. 2008), though still more than an order of magnitude lower than SGRB 060121 even at the lower of the two photometric redshift solutions quoted in de Ugarte Postigo et al. (2006).

There appears to be significant bias at the highest redshifts toward preferentially detecting the most luminous short bursts (see Figure 9). In particular, with the noted exception of SGRB 070429B, the highest redshift short bursts consistently have a burst luminosity higher than any lower redshift burst. Whether this trend of finding ever more luminous bursts as we probe ever higher redshifts is partially due to evolution in burst luminosity with redshift or solely the result of detector sensitivity limits and other selection effects remains to be determined. Regardless, considerable care must be exercised when studying the properties of high redshift short bursts and especially when comparing them with those of the local population.

SGRB 070714B, and also SGRB 070429B, firmly moves back the time at which we know short bursts were being formed into the first half of the age of the Universe (and further dispels the notion that short bursts require an old progenitor population). With further host identifications, it may become possible to observe evolution in the SGRB host galaxy types and possibly set observational constraints on the formation time required by the progenitor system. Indeed in the case of Type Ia supernovae, a lack of of type Ia's observed at $z>1.4$, and the suggested dearth thereof, has put considerable constraints on their formation time and models (Strolger et al. 2004; Dahlen et al. 2008). The ever-increasing redshift detections, and likely bias toward detection of only the more luminous short bursts at the highest redshifts, suggests that short bursts may well have occurred throughout nearly all the history of the Universe.

This paper is based on observations made with the Gemini North and South, KECK II, Liverpool, and William Herschel Telescopes.

We wish to thank $\mathrm{K}$. Roth and other members of the Gemini staff for their help in scheduling and implementing these observations. The Gemini Observatory is operated by the Association of Universities for Research in Astronomy, Inc., under a cooperative agreement with the NSF on behalf of the Gemini partnership: the National Science Foundation (United States), the Science and Technology Facilities Council (United Kingdom), the National Research Council (Canada), CONICYT (Chile), the Australian Research Council (Australia), Ministrio da Cincia e Tecnologia (Brazil), and SECYT (Argentina). Data were collected under programs GN-2007A-Q-28, GN-2007BQ-31, and GS-2007B-Q-12.

The W.M. Keck Observatory is operated as a scientific partnership among the California Institute of Technology, the University of California, and the National Aeronautics and Space Administration. The Observatory was made possible by the generous financial support of the W.M. Keck Foundation. The authors wish to recognize and acknowledge the very significant cultural role and reverence that the summit of Mauna Kea has always had within the indigenous Hawaiian community. We are most fortunate to have the opportunity to conduct observations from this mountain.

The Liverpool Telescope is operated on the island of $\mathrm{La}$ Palma by Liverpool John Moores University in the Spanish 
Observatorio del Roque de los Muchachos of the Instituto de Astrofisica de Canarias with financial support from the UK Science and Technology Facilities Council.

The William Herschel Telescope is operated on the island of La Palma by the Isaac Newton Group in the Spanish Observatorio del Roque de los Muchachos of the Instituto de Astrofisica de Canarias. The authors thank the overridden observes D. Bonfield and A. Sansigre for performing their ToO observations.

Facilities: Gemini:Gillett (GMOS, NIRI), Keck:II (NIRSPEC), Gemini:South (GMOS), Liverpool:2m, ING:Herschel

\section{REFERENCES}

Abraham, R. G., et al. 2003, Gemini Observatory Newsletter, 26, 6

Barbier, L., et al. 2007, GRB Coordinates Network, 6623

Barr, J., Davies, R., Jørgensen, I., Bergmann, M., \& Crampton, D. 2005, AJ, 130,445

Belczynski, K., Hartmann, . H., Fryer, C. L., Holz, D. E., \& O’Shea, B. 2008, ApJ, submitted (arXiv:0812.2470)

Belczynski, K., Perna, R., Bulik, T., Kalogera, V., Ivanova, N., \& Lamb, D. Q. 2006, ApJ, 648, 1110

Berger, E. 2009, ApJ, 690, 231

Berger, E., et al. 2005, Nature, 438, 988

Berger, E., et al. 2007, ApJ, 664, 1000

Blinnikov, S. I., Novikov, I. D., Perevodchikova, T. V., \& Polnarev, A. G. 1984, Sov. Astron. Lett., 10, 177

Bloom, J. S., Frail, D. A., \& Sari, R. 2001, AJ, 121, 2879

Bloom, J. S., et al. 2006, ApJ, 638, 354

Bloom, J. S., et al. 2009, ApJ, 691, 723

Böhm, A., et al. 2004, A\&A, 420, 97

Cenko, S. B., et al. 2008, ApJ, submitted (arXiv:0802.0874)

Christensen, L., Hjorth, J., \& Gorosabel, J. 2004, A\&A, 425, 913

Cobb, B. E., \& Bailyn, C. D. 2008, ApJ, 677, 1157

Coleman, G. D., Wu, C.-C., \& Weedman, D. W. 1980, ApJS, 43, 393

Cuillandre, J. C., et al. 1994, A\&A, 281, 603

Dahlen, T., Strolger, L.-G., \& Riess, A. G. 2008, ApJ, 681, 462

Davies, M. B., Levan, A. J., \& King, A. R. 2005, MNRAS, 356, 54

de Ugarte Postigo, A., et al. 2006, ApJ, 648, L83

Dermer, C. D., \& Atoyan, A. 2006, ApJ, 643, L13

Dimitrijević, M. S., Popović, L. Č., Kovačević, J., Dačić, M., \& Ilić, D. 2007, MNRAS, 374, 1181

Donaghy, T. Q., et al. 2006, ApJ, submitted (astro-ph/0311045)

Eichler, D., Livio, M., Piran, T., \& Schramm, D. N. 1989, Nature, 340, 126

Fox, D. B., et al. 2005, Nature, 437, 845

Fruchter, A. S., et al. 1999, ApJ, 519, L13

Fruchter, A. S., et al. 2006, Nature, 441, 463

Gehrels, N., et al. 2004, ApJ, 611, 1005

Gehrels, N., et al. 2005, Nature, 437, 851

Glazebrook, K., \& Bland-Hawthorn, J. 2001, PASP, 113, 197

Glazebrook, K., \& the GDDS team. 2003, astro-ph/0311045

Goodman, J. 1986, ApJ, 308, L47

Graham, J. F., Fruchter, A. F., Levan, A. J., Nysewander, M., Tanvir, N. R.,

Dahlen, T., Bersier, D., \& Pe'er, A. 2007, GRB Coordinates Network, 6836 Greiner, J., et al. 2009, ApJ, 693, 1610

Gwyn, S. D. J. 1995, MS thesis, Univ. of Victoria

Hjorth, J., et al. 2003, Nature, 423, 847

Hjorth, J., et al. 2005a, ApJ, 630, L117

Hjorth, J., et al. 2005b, Nature, 437, 859

Jester, S., et al. 2005, AJ, 130, 873
Jørgensen, I., Bergmann, M., Davies, R., Barr, J., Takamiya, M., \& Crampton, D. 2005, AJ, 129, 1249

Kann, D. A., Masetti, N., \& Klose, S. 2007, AJ, 133, 1187

Kann, D. A., et al. 2008, ApJ, submitted (arXiv:0804.1959)

Katz, J. I., \& Canel, L. M. 1996, ApJ, 471, 915

Kewley, L. J., \& Dopita, M. A. 2002, ApJS, 142, 35

Kinney, A. L., Calzetti, D., Bohlin, R. C., McQuade, K., Storchi-Bergmann, T., \& Schmitt, H. R. 1996, ApJ, 467, 38

Kouveliotou, C., Meegan, C. A., Fishman, G. J., Bhat, N. P., Briggs, M. S., Koshut, T. M., Paciesas, W. S., \& Pendleton, G. N. 1993, ApJ, 413, L101

Landolt, A. U. 1992, AJ, 104, 340

Landsman, W., Marshall, F. E., \& Racusin, J. 2007, GRB Coordinates Network, 6689

Landsman, W., \& Racusin, J. 2007, GRB Coordinates Network, 6632

Le Floc'h, E., Charmandaris, V., Forrest, W. J., Mirabel, I. F., Armus, L., \& Devost, D. 2006, ApJ, 642, 636

Le Floc'h, E., Mirabel, I. F., \& Duc, P.-A. 2002, JA, 23, 119

Le Floc'h, E., et al. 2003, A\&A, 400, 499

Lee, W. H., Ramirez-Ruiz, E., \& Granot, J. 2005, ApJ, 630, L165

Levan, A. J., Tanvir, N. R., Bonfield, D., Martinez-Sansigre, A., Graham, J., \& Fruchter, A. 2007b, GRB Coordinates Network, 6630

Levan, A. J., Wynn, G. A., Chapman, R., Davies, M. B., King, A. R., Priddey, R. S., \& Tanvir, N. R. 2006c, MNRAS, 368, L1

Levan, A. J., et al. 2006a, GRB Coordinates Network, 4841

Levan, A. J., et al. 2006b, ApJ, 648, L9

Levan, A. J., et al. 2007a, MNRAS, 378, 1439

Mazets, E. P., et al. 1981, Ap\&SS, 80, 3

Melandri, A. 2007, GRB Coordinates Network, 6621

Mobasher, B., Rowan-Robinson, M., Georgakakis, A., \& Eaton, N. 1996, MNRAS, 282, L7

Modjaz, M., et al. 2008, AJ, 135, 1136

Nakar, E., Gal-Yam, A., \& Fox, D. B. 2006, ApJ, 650, 281

Norris, J. P., Barthelmy, S. D., \& N., G. 2005, GRB Coordinates Network, 4384

Norris, J. P., Cline, T. L., Desai, U. D., \& Teegarden, B. J. 1984, Nature, 308, 434

Nysewander, M., Fruchter, A. S., \& Pe'er, A. 2008, ApJ, in press (arXiv:0806.3607)

Ohno, M., et al. 2007, GRB Coordinates Network, 6638

Paczynski, B. 1986, ApJ, 308, L43

Perley, D. A., Bloom, J. S., Modjaz, M., Poznanski, D., \& Thoene, C. C. 2007, GRB Coordinates Network, 7140

Piranomonte, S., et al. 2008, A\&A, 491, 183

Prochaska, J. X., Weiner, B. J., Chen, H.-W., \& Mulchaey, J. S. 2006, ApJ, 643, 680

Racusin, J. L., Barthelmy, J. L., Burrows, N. D., Chester, M. M., Gehrels, N., Krimm, H. A., Palmer, D. M., \& Sakamoto, T. 2007b, GRB Coordinates Network, 6620

Racusin, J. F., Kennea, J., Pagani, C., Vetere, L., \& Evans, P. 2007a, GRB Coordinates Network, 6627

Schlegel, D. J., Finkbeiner, D. P., \& Davis, M. 1998, ApJ, 500, 525

Schmidt, M., Higdon, J. C., \& Hueter, G. 1988, ApJ, 329, L85

Seto, N. 2005, astro-ph/0512212

Soderberg, A. M., et al. 2006, ApJ, 650, 261

Stanek, K. Z., et al. 2003, ApJ, 591, L17

Stanek, K. Z., et al. 2007, ApJ, 654, L21

Storey, P. J., \& Zeippen, C. J. 2000, MNRAS, 312, 813

Stratta, G., et al. 2007, A\&A, 474, 827

Strolger, L.-G., et al. 2004, ApJ, 613, 200

Usov, V. V. 1992, Nature, 357, 472

Vreeswijk, P. M., et al. 2007, A\&A, 468, 83

Williams, R. E., et al. 1996, AJ, 112, 1335

Woosley, S. E., \& Bloom, J. S. 2006, ARA\&A, 44, 507

Zhang, B., et al. 2009, ApJ, submitted (arXiv:0902.2419) 